\title{
Human evolution has its Dobzhansky moment
}

"Just as the phenomenon of mutation did not become visible to Morgan until his breeding experiments crossed a certain threshold of scale, so, too, Dobzhansky and Sturtevant hit a critical threshold of scale after which they began to perceive the distinctive genetic patterns of regional populations." Robert E. Kohler in Lords of the Fly (University of Chicago Press, 1994).

umans shuffle uneasily on the catwalk of model organisms. The yeasts are there for their genomic minimalism. The worm gets a lot of function from its few neurons. The puffer fish proudly displays its tiny introns, and the snapdragon has snipped seasons of work from an unstable set of mutations. In contrast, the reasons we stand to be scrutinized range from the medically urgent to the frankly narcissistic. We're there because we have a self-selecting screen, because of the excellence of human phenotyping, for interest in the mutations that afflict us and also, well, because of our curiosity about ourselves. So, although we expect the model organisms to be the first to deliver results that fit us too, it can be upsetting to see just how early some of these first discoveries were. Is it really possible that a result obtained in a fly by genetics pioneers Theodosius Dobzhansky and Alfred Sturtevant more than 70 years ago has only just been found to apply to us?

In this issue, Hreinn Stefansson and colleagues (p 129) describe a polymorphic chromosomal inversion that changed in frequency with geographic location and is locally under ongoing selection for fertility. Both these authors and Arcadi Navarro and Elodie Gazave (News and Views p 115) emphasize parallels between this work and the classic investigation into the population genetics of chromosome polymorphisms in the fly, Drosophila pseudoobscura. But can it really be so simple? Apply a historically productive strategy from a model system at the right time, and the secrets of human evolution will yield to population genetics analysis?

Maybe the real lesson from history is the choice of the scale of investigation (as Robert Kohler's quote above suggests) and the timely application of appropriate technology. There was a 17-year gestation period from Sturtevant's publication identifying chromosome rearrangements from the disturbance they make to the Drosophila melanogaster linkage map (Publ. Carnegie Inst. 278, 305-341; 1919) to the routine use of inversion polymorphisms in $D$. pseudoobscura for evolutionary population genetics by Sturtevant and Dobzhansky (Proc. Natl. Acad. Sci. USA 22, 448-450; 1936 and Genetics 23, 28-64; 1938). Part of this time was the wait for a suitably rapid technique for cytological visualization of inversions, replacing the protracted procedure of testing wild-caught chromosomes by linkage mapping against a laboratory reference strain.

Judging by the pages of Nature Genetics, there has been an 8-year gap from the recognition of a human inversion polymorphism to the use of such a rearrangement in evolutionary population genetics. Kersten Small and colleagues (Nat. Genet. 16, 96-99; 1997) first showed that a polymorphic inversion was present in a large proportion of the population without any associated disease. These authors noted that this $48-\mathrm{kb}$ inversion contributes a reduction in genetic map distance and a region of extended linkage disequilibrium to a region of the $\mathrm{X}$ chromosome, and they speculated that similar rearrangements might be a general feature of the human genome that would be revealed by long range genomic sequencing. This intervening period has seen an explosion of genome sequencing and genotyping technology and its application to genetic disease and to human evolution.

Another lesson of history is to tackle the right problem with the right experimental tools and paradigm. Dobzhansky was able to survey a large number of local populations because cytologically visible inversions were easy to recognize. But his drive to demonstrate evolution experimentally was the other key to his success. The same ease of recognition led others to investigations of these markers in many controlled lab environments and in a great range of biological phenomena discussed in the News and Views ( $p$ 115). In the current research era, genome sequencing strategies have delivered a bonanza of SNPs that are being used in many different studies. Perhaps it was not the most obvious application to use SNPs to examine the evolutionary machinations of structural rearrangements, but innovation can yield great rewards.

Inversions don't exactly jump out of the human genome. Although structural rearrangements can be inferred from linkage disequilibrium or recombination patterns, Hreinn Stefansson and colleagues needed several additional steps to confirm the presence and population-level effects of the inversion. Empirical SNP data derived from BAC clones were used to establish the long-range haplotypes and inversion structure. Demographic simulations were used to examine frequency changes that would be likely with and without selection. And it took an unusually large, deep and welldocumented pedigree set to demonstrate currently operating selection.

Even among flies, it is not clear that inversions are key agents of evolutionary change. They are excellent markers, but do they indicate where the evolutionary action is occurring in the genome? The early geneticists found that the importance of inversions varies with the ecology of the beasts that bear them; those inhabiting a diverse lot of local environments had more polymorphic inversions, possibly harboring complexes of coadapted alleles. It will be interesting to see whether, in our genome polymorphism, humans most closely resemble niche-specializing $D$. pseudoobscura or whether we are more like our cosmopolitan commensal, D. melanogaster. 